\title{
Eosinophilic Gastrointestinal Disorder in Coeliac Disease: A Case Report and Review
}

\author{
Dennis N. F. Lim, Annelise Wilkins, Laura Elizabeth Horton, Ishfaq Ahmad, \\ Catherine lo Polito, and Chris Phillips
}

Department of Gastroenterology, Alexandra Hospital, Worcestershire Acute Hospitals NHS Trust, Woodraw Drive, Redditch B98 7UB, UK

Correspondence should be addressed to Dennis N. F. Lim, dennis2020@yahoo.com

Received 3 November 2012; Accepted 22 November 2012

Academic Editors: P. Abraham, O. I. Giouleme, A. Imagawa, S. Nomura, C. T. Shun, and Ö. Yönem

Copyright ( $) 2012$ Dennis N. F. Lim et al. This is an open access article distributed under the Creative Commons Attribution License, which permits unrestricted use, distribution, and reproduction in any medium, provided the original work is properly cited.

\begin{abstract}
Eosinophilic gastrointestinal disorder is a rare disorder characterised by eosinophilic infiltration of the gastrointestinal tract. There are various gastrointestinal manifestations with eosinophilic ascites being the most unusual and rare presentation. Diagnosis requires high index of suspicion and exclusion of various disorders associated with peripheral eosinophilia. There are no previous case reports to suggest an association between eosinophilic gastrointestinal disorder and coeliac disease in adults. We report a case of eosinophilic ascites and gastroenteritis in a 30-year-old woman with a known history of coeliac disease who responded dramatically to a course of steroids.
\end{abstract}

\section{Introduction}

Eosinophilic gastrointestinal disorder is an unusual inflammatory gastrointestinal disease characterized by eosinophilic gut infiltration [1]. There have been case series and cohort studies suggesting an association between eosinophilic oesophagitis and coeliac disease in children. However, there were no previous reports or studies to indicate an association between coeliac disease and eosinophilic gastrointestinal disorder in adults. This paper examines a patient with concurrent eosinophilic gastrointestinal disorder and coeliac disease.

\section{Case Report}

A 30-year-old Caucasian female presented with a two-week history of central abdominal pain and distension, vomiting, and increased frequency of bowel motion. There had been no weight loss or history of foreign travel. She was known to have coeliac disease diagnosed at age two. She was compliant to gluten-free diet. There was no history of drug allergy, asthma or skin eczema. Abdominal examination showed sign of ascites. There were no stigmata of chronic liver disease. The rest of the systemic examination was also within normal limits. A summary of her laboratory investigations is presented in Table 1. Computed tomography of the abdomen and pelvis showed large volume ascites and prominent small bowel loops (Figures 1(a) and 1(b)). There was no evidence of a pelvic or peritoneal mass. A diagnostic paracentesis revealed straw-coloured ascites. Analysis of the ascitic fluid is summarized in Table 1. Oesophagogastroduodenoscopy (Figure 2) showed multiple areas of erythematous patches at the superior duodenal angle close to the second portion of the duodenum. Colonoscopy and biopsies of colonic and terminal ileal mucosa were normal. Histological examination of duodenal mucosa revealed Marsh 3a villous atrophy consistent with coeliac disease although no previous biopsies were available for comparison (Figure 3 ).

Jejunal mucosa showed dense infiltration of eosinophils on the lamina propria (30/HPF) (Figures 4 and 5). There was no histological evidence of an enteropathy-associated T-cell lymphoma, granuloma, or malignancy. Unfortunately, the endoscopist did not obtain oesophageal and gastric biopsies.

Laporoscopic examination showed no evidence of underlying intra-abdominal malignancy. After excluding the possibilities of malignancy, parasitic disease and an autoimmune 
TABLE 1: Summary of laboratory results.

Peripheral eosinophil count

$860 \mathrm{IU} / \mathrm{L}$

$\operatorname{IgE}$

(normal 12-760 IU/L)

$98 \mathrm{IU} / \mathrm{L}$

(normal 13-128 IU/L)

Negative 36.4 IU/L

(normal $=$ negative $)$

Negative

(normal $=$ negative $)$

Negative

Negative for oval, cysts and parasites

Negative

(Echinococcus,

Entamoeba histolytica, Toxocara,

Trichinella)

RAST (radioallergosorbent test) egg, peanut, soya, milk, cheese, wheat, cod, mussel, salmon, shrimp, tuna, hazel, Brazil, and almond nuts

Ascitic fluid analysis

Total protein

Albumin

SAAG (serum ascitic-albumin gradient)

Eosinophil count

Cytology

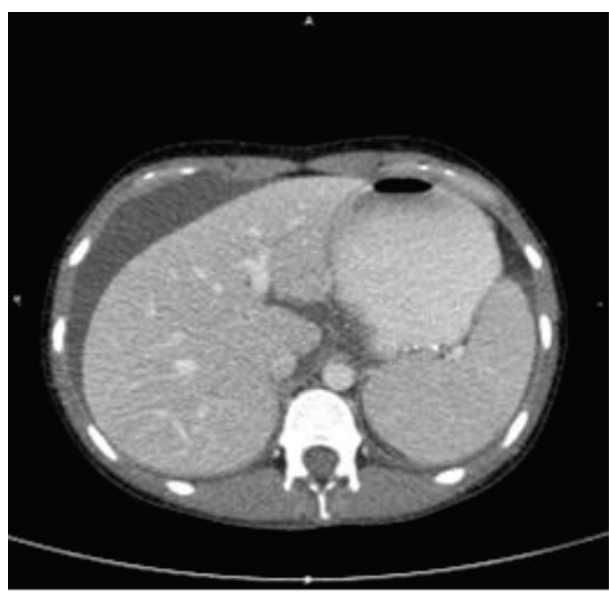

(a)

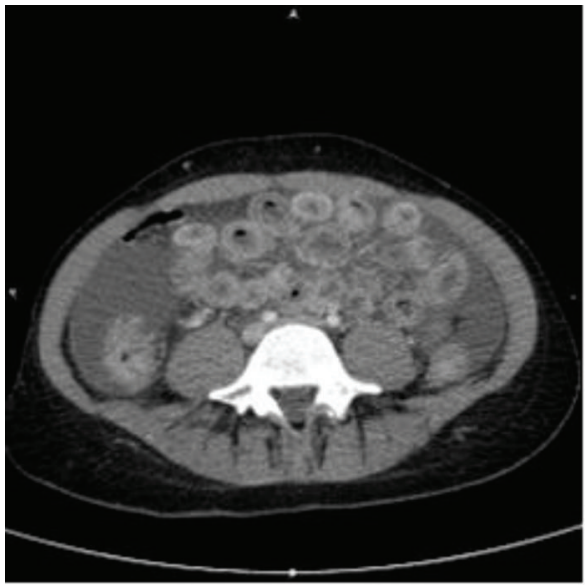

(b)

FIGURE 1: Computed tomography of abdomen and pelvis showing large amount of ascites and thickened small bowel wall. No pelvis or peritoneal mass identified.

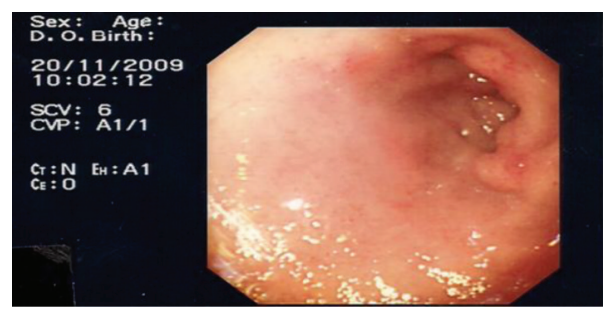

FIGURE 2: Esophagogastroduodenoscopy showed multiple areas of erythematous patches at the duodenal bulb.

layers of the intestinal wall: the mucosa, muscle, and subserosal layers [1]. Depending upon the area involved, the disease can present as eosinophilic oesophagitis, eosinophilic gastroenteritis, eosinophilic ascites, and eosinophilic colitis.

In a retrospective study of 40 patients, the most common symptoms were abdominal pain, nausea, vomiting, and diarrhoea. In this study, the percentages involving mucosal layer, muscle, and subserosal layers were 58\%, 30\%, and $12 \%$ respectively [3]. described in 1937 [2]. The disorder is classified according to the predominance of eosinophilic infiltration in different 


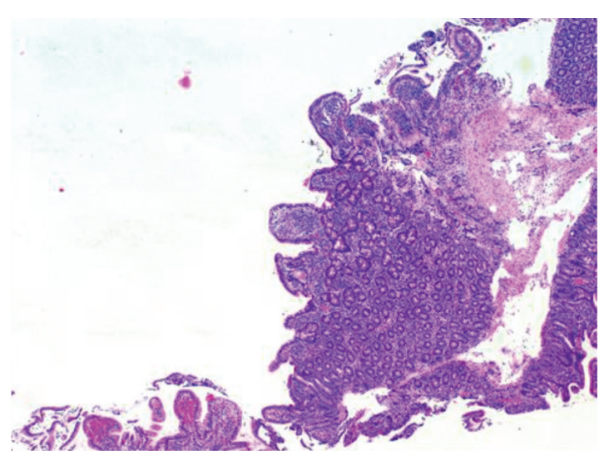

Figure 3: H\&E Low power view of second part of duodenal biopsy showing Marsh 3a villous atrophy.

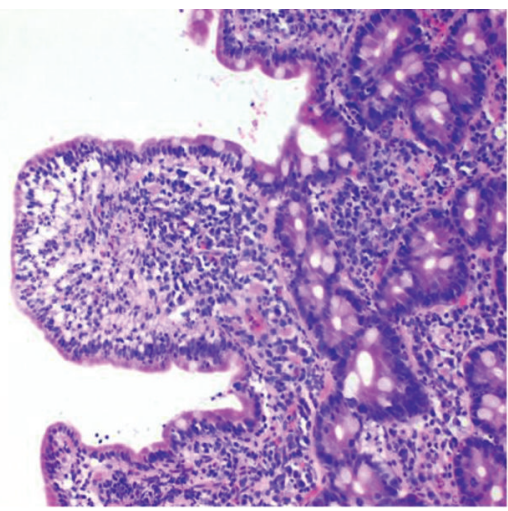

FIGURE 4: H\&E high power view of jejunal biopsy showed increase in lamina propria cellularity with eosinophilic infiltration.

Radiological features of eosinophilic gastroenteritis include thickening of mucosal folds on barium study or computed tomography depending on the severity and layer(s) involved [4]. However, similar changes may also be seen in Menetrier's disease, lymphoma, and Crohn's and granulomatous disease. Therefore, the thickening of bowel wall is not specific for eosinophilic gastroenteritis. The endoscopic appearance in eosinophilic gastroenteritis is also nonspecific, including erythematous, friable, nodular, and occasional ulcerative changes [5]. Mucosal biopsies are nondiagnostic in approximately $10 \%$ of cases either because of sampling error or patchy involvement. Therefore, a minimum of eight biopsies is recommended to establish the diagnosis $[3,6,7]$.

Both cellular and humoral immunity have been postulated placing eosinophilic gastroenteritis between purely IgE-mediated disorders such as food allergies and non-IgEmediated disorders such as inflammatory bowel disease and coeliac disease $[8,9]$. It is known that activated eosinophils are found within the lamina propria of patients with coeliac disease, especially in the later stages after lymphocyte activation. These eosinophils have the capacity to synthesise IL-5, a cytokine produced by $\mathrm{T}$ and mast cells, which is the major actor involved in eosinophils differentiation and activation [8]. Although a subset of these patients has an allergic etiology and mucosal disease; there is a good deal

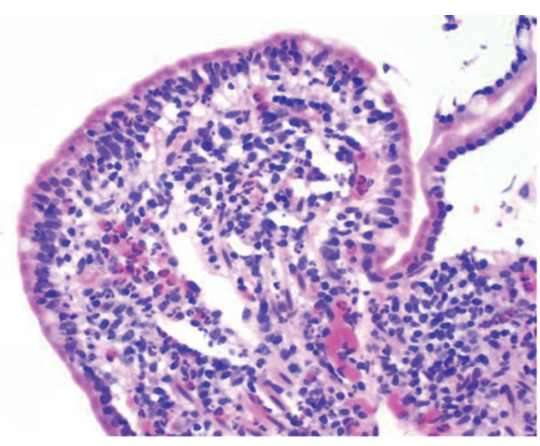

Figure 5: H\&E section of jejunal biopsy showed eosinophils infiltration $(30 / \mathrm{HPF})$ in the lamina propria.

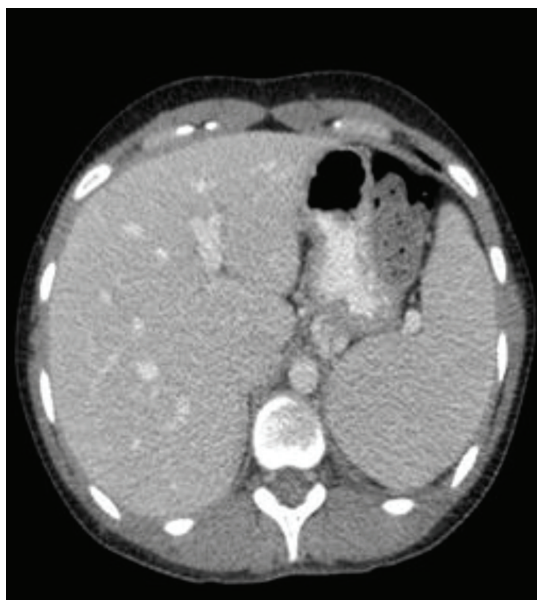

(a)

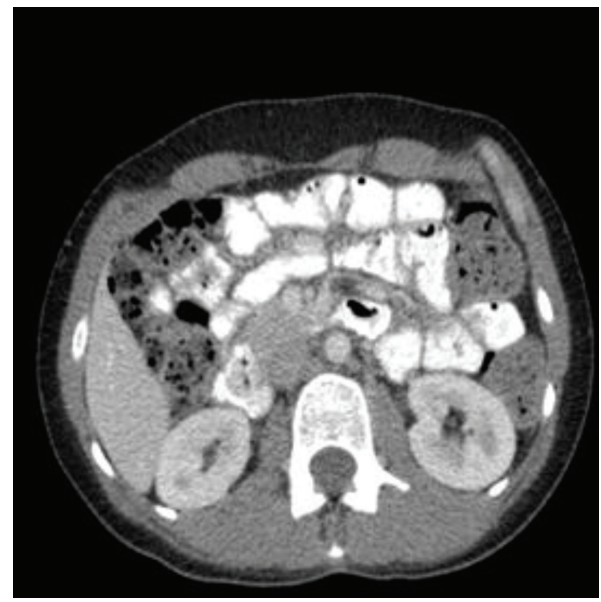

(b)

FIGURE 6: Computed tomography showing resolution of ascites and small bowel thickening three months after discharge from hospital.

of heterogeneity in the disease in regard to both allergic sensitization and anatomic location. Histopathological examination of the biopsies and ascetic fluid analysis are essential parts of the diagnostic workup.

The patient in our case report has a childhood history of coeliac disease at the age of two from another hospital. 
The diagnosis was based on gastrointestinal symptoms of ascites and gastroenteritis, the presence of dense eosinophil infiltration on the lamina propria of duodenal and jejunal biopsies, marked eosinophilia in the ascites, and the rapid response to steroid therapy [3]. However, unlike eosinophilic oesophagitis, there is no consensus definition of eosinophilic gastroenteritis or well-accepted cutoff eosinophil number per high power field. A large proportions of patients diagnosed with eosinophilic gastroenteritis responded to oral steroid in a dramatic fashion $[1,3]$. The appropriate duration of steroid treatment remains unknown but improvement usually occurs within two weeks regardless of the layer of bowel involved [10]. Some patients may require prolonged therapy to induce resolution of symptoms [10]. In a small proportion of patients with a relapsing-remitting course, long-term low-dose steroids or immunosuppressive therapy may be required. A novel approach with the use of leukotriene receptor antagonists (Montelukast) has been successfully used in treatment of serosal eosinophilic gastroenteritis as steroidsparing therapy [11]. This treatment is important especially in the paediatric patients. In addition, the use of monoclonal antibodies against IgE and IL5 such as omalizumab and mepolizumab, respectively, has shown promising results in the treatment of patients with eosinophilic gastroenteritis in clinical trials $[9,12]$.

\section{Conclusions}

Elevated eosinophils in blood and different tissues have been found in coeliac disease. The coexistences of eosinophilic gastrointestinal disorder and coeliac disease in an adult should be considered when a patient presented with ascites in the absence of liver disease and unexplained gastrointestinal symptoms.

\section{References}

[1] N. C. Klein, R. L. Hargrove, M. H. Sleisenger, and G. H. Jeffries, "Eosinophilic gastroenteritis," Medicine, vol. 49, no. 4, pp. 299-319, 1970.

[2] R. Kaijser, "Zur Kenntnis der allergischen affektionen des verdauungskanals vom standpunkt des chirurgen aus," Archiv für Klinische Chirurgie, vol. 188, pp. 36-64, 1937.

[3] N. J. Talley, R. G. Shorter, S. F. Phillips, and A. R. Zinsmeister, "Eosinophilic gastroenteritis: a clinicopathological study of patients with disease of the mucosa, muscle layer, and subserosal tissues," Gut, vol. 31, no. 1, pp. 54-58, 1990.

[4] R. L. MacCarty and N. J. Talley, "Barium studies in diffuse eosinophilic gastroenteritis," Gastrointestinal Radiology, vol. 15, no. 3, pp. 183-187, 1990.

[5] V. L. Fox, "Eosinophilic esophagitis: endoscopic findings," Gastrointestinal Endoscopy Clinics of North America, vol. 18, pp. 45-57, 2008.

[6] A. J. Katz, H. Goldman, and R. J. Grand, "Gastric mucosal biopsy in eosinophilic (allergic) gastroenteritis," Gastroenterology, vol. 73, no. 4, pp. 705-709, 1977.

[7] M. Lee, W. G. Hodges, T. L. Huggins, and E. L. Lee, "Eosinophilic gastroenteritis," Southern Medical Journal, vol. 89, no. 2, pp. 189-194, 1996.
[8] M. E. Rothenberg, "Eosinophilic gastrointestinal disorders (EGID)," Journal of Allergy and Clinical Immunology, vol. 113, no. 1, pp. 11-29, 2004.

[9] S. Foroughi, B. Foster, N. Kim et al., "Anti-IgE treatment of eosinophil-associated gastrointestinal disorders," Journal of Allergy and Clinical Immunology, vol. 120, no. 3, pp. 594-601, 2007.

[10] C. M. Lee, C. S. Changchien, P. C. Chen et al., "Eosinophilic gastroenteritis: 10 Years experience," American Journal of Gastroenterology, vol. 88, no. 1, pp. 70-74, 1993.

[11] M. C. Urek, M. Kujundžić, M. Banić, R. Urek, T. Š. Veić, and D. Kardum, "Leukotriene receptor antagonists as potential steroid sparing agents in a patient with serosal eosinophilic gastroenteritis," Gut, vol. 55, no. 9, pp. 1363-1364, 2006.

[12] M. E. Rothenberg, A. D. Klion, F. E. Roufosse et al., "Treatment of patients with the hypereosinophilic syndrome with mepolizumab," New England Journal of Medicine, vol. 358 , no. 12, pp. 1215-1228, 2008. 


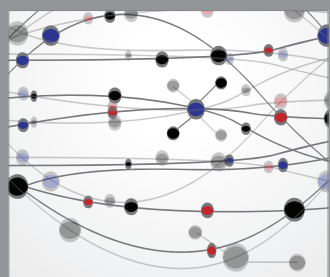

The Scientific World Journal
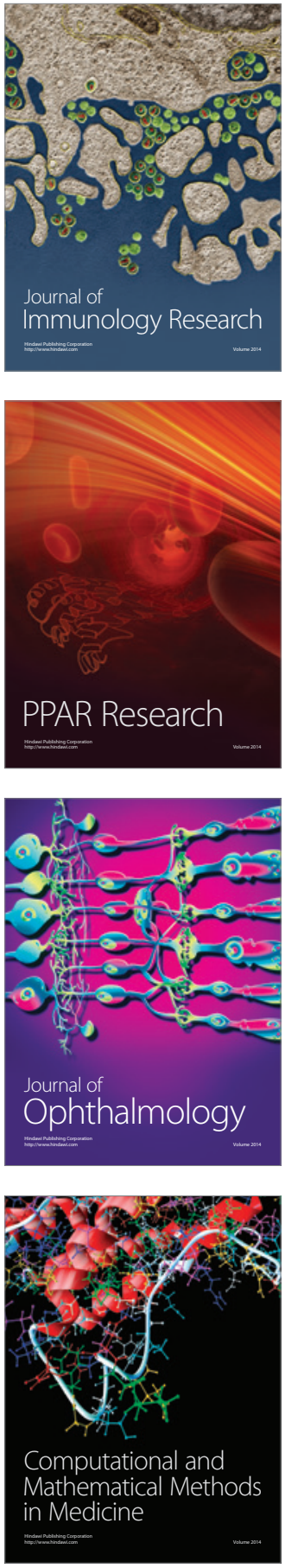

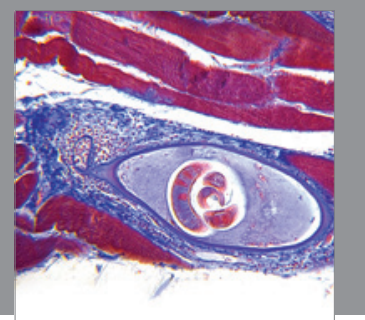

Gastroenterology

Research and Practice
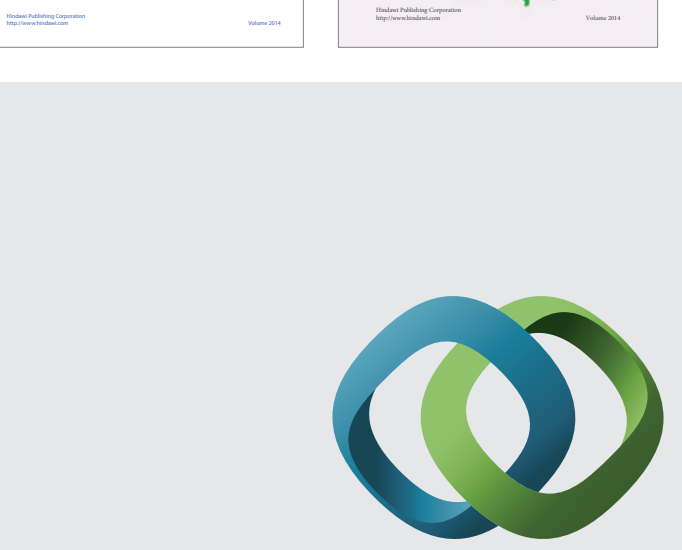

\section{Hindawi}

Submit your manuscripts at

http://www.hindawi.com
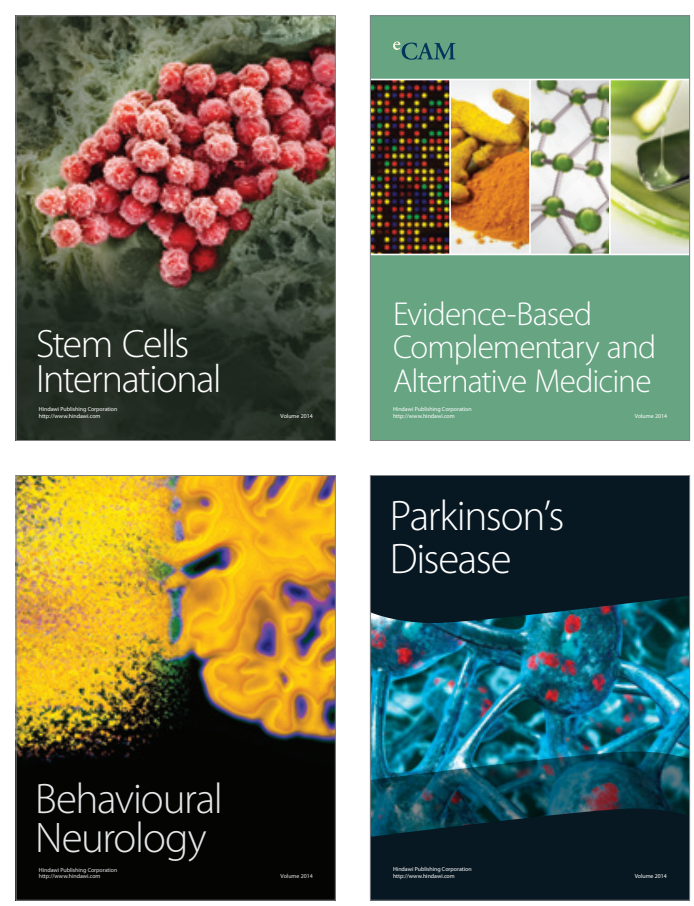

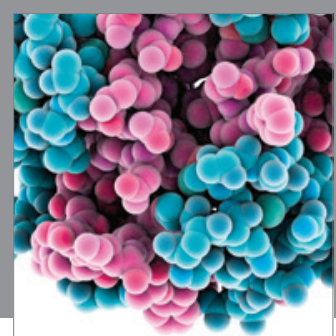

Journal of
Diabetes Research

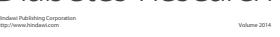

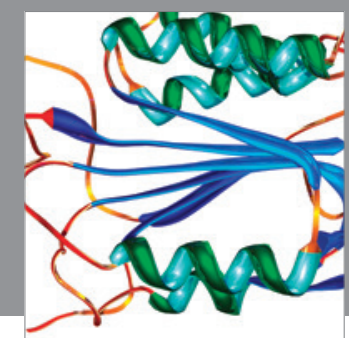

Disease Markers
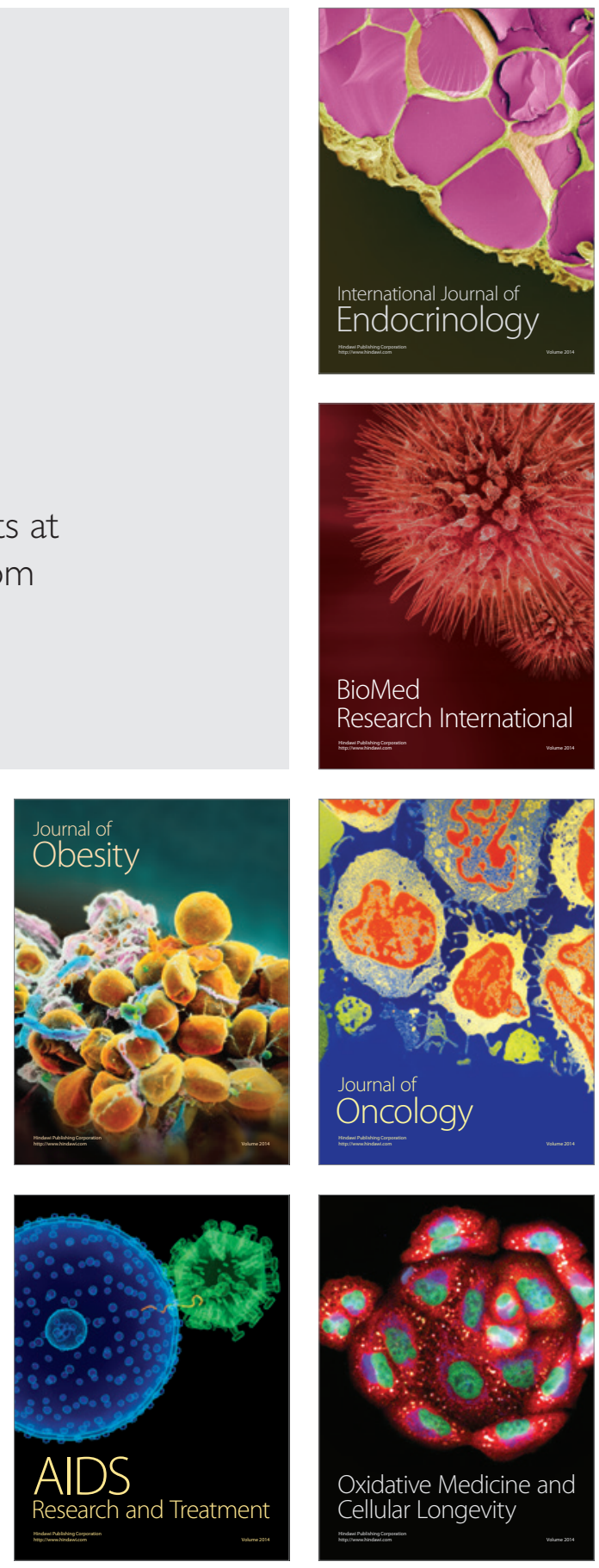